\title{
Manual Sutureless Cataract Surgery (MSCS) in Patients with Pseudo-Exfoliation
}

\author{
Yasir lqbal ${ }^{1}$, Qaim Ali Khan ${ }^{2}$, Sohail Zia ${ }^{3}$, Muhammad Usman Arshad Qureshi ${ }^{4}$, Masud-ul-Hassan ${ }^{5}$ \\ ${ }^{1}$ Watim Medical College, Rawat, Rawalpindi \\ ${ }^{2}$ Poonch Medical College, Rawalakot, ${ }^{3-5}$ Ripah International University, Islamabad
}

\begin{abstract}
Purpose: To document the complications of Manual suture-less cataract surgery in eyes with pseudo-exfoliation.

Study Design: Interventional case series.

Place and Duration of Study: Naseer memorial hospital, Dadyal, Azad Jammu Kashmir, from 4 July 2017 to 3 July 2019.

Methods: One hundred and fifty patients with pseudo-exfoliation (PEX) and cataract were selected by convenient sampling. Patients with systemic diseases, history of trauma, intraocular pressure $\geq 15 \mathrm{~mm} \mathrm{Hg}$ on Applanation tonometry and any other associated ocular disease for example retinal detachment or retinal disease, previous history of glaucoma or narrow/closed angle on Gonioscopy were excluded from the study. Dark brown cataracts were also excluded. Pseudo-exfoliation was diagnosed on slit lamp on the basis of presence of dandruff like material on the pupil and the anterior lens capsule. The patients underwent manual suture less cataract surgery under local anesthesia Intraoperative and post-operative complications were noted. first day postoperatively. The collected data and analyzed using statistical package for social sciences (SPSS) version 21 for analysis.

Results: The study group consisted of $64 \%$ males and $36 \%$ females with mean age of $65 \pm 15.32$ years. The most common difficulty encountered was poor pupil dilatation in $37.3 \%$ cases followed by Zonular dehiscence in $7.3 \%$. On the first post-operative day, the most common complication was intraocular lens decentration in $2.6 \%$ of the cases.

Conclusion: Pupils of patients with pseudo-exfoliation dilate poorly and makes surgery difficult. With good pupillary dilatation, careful capsulorhexis and minimal stress on the zonules, MSCS can be safely performed in eyes with cataract and PEX.
\end{abstract}

Key Words: Pseudo-exfoliation, Cataract, Pupil.

How to Cite this Article: lqbal Y, Khan QA, Qureshi MUA, Hassan M. Manual Sutureless Cataract Surgery (MSCS) in Patients with Pseudo-Exfoliation. Pak J Ophthalmol. 2020; 36 (4): 391-395.

Doi: https://doi.org/10.36351/pjo.v36i4.1097

\section{INTRODUCTION}

Pseudo-exfoliation (PEX) is an ocular condition in which a grey white fibrillar material appears on the

Correspondence: Yasir Iqbal

Department of Ophthalmology Watim Medical College, Rawat, Rawalpindi

Email: yazeriqbal@gmail.com

Received: July 8, 2020

Accepted: August 18, 2020 pupil, anterior lens surface, trabecular meshwork and anterior hyloid. PEX is an extracellular material produced by abnormal aging cells epithelium and has been seen in other visceral organs and skin which suggests that it is a systemic disease. ${ }^{1}$ Deposition of PEX material leads to clogging of the trabecular meshwork which causes increase in intraocular pressure and secondary open angle glaucoma. ${ }^{2}$

Globally the prevalence of PEX is variable i.e. Ethiopia 39.3\%, Greece 28\%, Turkey $16.7 \%$, Indian 
occupied Kashmir 26.3\% and in Pakistan it is reported to be $8 \%{ }^{3}$ PEX is usually present in the old age group and it is the old age group in which cataract is also coomon. ${ }^{4}$

Cataract surgery is the most common ocular surgery being performed in all ophthalmic departments. Recent estimates are that 94 million people are visually impaired and 20 million are blind due to cataract worldwide and this number is increasing with every passing day. ${ }^{5}$ Cataract can be normal age related or it can secondary either due to trauma, drugs, ocular causes or systemic diseases. Whatever the cause, the only available treatment of cataract is surgery with implantation of intraocular lens.

Ophthalmic surgery and especially cataract surgery is undergoing advancement day by day. Conventional extracapsular cataract extraction (ECCE) has become almost obsolete and surgeons have shifted to Phacoemulsification and manual sutureless cataract surgery (MSCS). ${ }^{6}$ Phacoemulsification has a good quick visual recovery time, less intraocular inflammation and less postoperative astigmatism and is normally present in all tertiary care hospitals in the western world. The technique of MSCS, although developed much later than Phacoemulsification, has become a frequent procedure for cataract surgery in the developing world because of its cost effectiveness and low socioeconomic status of the masses. ${ }^{6}$

Techniques of cataract surgery are being customized in patients with PEX but no particular technique has been demonstrated to be completely safe and without complications. Limited information exists about problems and complications of performing MSCS in PEX. Therefore, we aimed to document complications of performing MSCS in eyes with PEX.

\section{METHODS}

It was an interventional case series conducted at Naseer memorial hospital, Dadyal, Azad Jammu Kashmir during period of two years from 4 July 2017 to 3 July 2019 . The study was conducted according to the guidelines of Declaration of Helsinki. The patients were allotted the medical registration number before the initiation of study. They underwent complete eye examination including visual acuity assessment, pupil examination, slit lamp examination, intraocular pressure measurement and detailed fundoscopy. One hundred and fifty patients were selected by using convenient sampling. PEX was diagnosed on slit lamp on the basis of presence of fibrin like material on the pupil of the lens surface. The inclusion criterion was any age and gender, presence of pseudo-exfoliation along with cataract and intraocular pressure $\leq 14 \mathrm{~mm}$ of $\mathrm{Hg}$. Exclusion criteria were systemic disease, history of trauma, intraocular pressure $\geq 15 \mathrm{~mm} \mathrm{Hg}$ on Applanation tonometry and any associated ocular disease like retinal detachment or retinal disease, previous history of glaucoma or narrow/closed angle on gonioscopy. Dark brown cataracts were also excluded because of more chances of per-operative complications. The operative procedure and its complications were explained to the patients before the surgery and they signed written consent form. Demographic information and eye to be operated were recorded. The patients underwent manual sutureless cataract surgery under local anesthesia by a single surgeon having expertise in the procedure.

One hour before the start of the surgery, Tropicamide $2 \%$ and Phenylephrine eye drops were instilled. Pupillary dilatation was measured under an operating microscope with a caliper. Any pupillary dilatation $<5 \mathrm{~mm}$ was considered to be poor pupillary dilatation. Before the initiation of the surgery, 5\% iodine was instilled in the conjunctival sac after draping the eye. At the temporal side the conjunctiva was undermined while cauterizing the bleeding vessels. Using a $3.2 \mathrm{~mm}$ keratome, a partial thickness 6-6.5 mm curved scleral incision was made $2 \mathrm{~mm}$ behind the limbus. A tunnel was constructed and was extended $1 \mathrm{~mm}$ into the corneal stroma. An internal corneal incision was made for entry into the anterior chamber and was extended $0.5 \mathrm{~mm}$ more than the external incision. Capsulorhexis was done with the help of a cystitome made by bending 1cc syringe and filling of anterior chamber with viscoelastic substance. Nucleus was rotated, picked up by the cystitome, displaced into the anterior chamber and expressed through the corneoscleral tunnel with the help of viscoelastic. Lens matter was removed by Irrigation/aspiration cannula and intraocular lens was implanted. A sub-conjunctival injection of antibiotic/ steroid was given and the eye was padded for 24 hours. Intraoperative complications, if any, were documented on the proforma and the surgical technique was modified according to the complication. The patients were followed up on the first postoperative day and complications, if any, were recorded on the proforma. Data was analyzed using statistical 
package for social sciences (SPSS) version 21 for analysis.

\section{RESULTS}

During the period of 2 years, 2457 patients were diagnosed and advised for cataract surgery. PEX was found in 8\% (197 cases). In 78\% cases, it was bilateral. PEX was found on Pupil in $72 \%$ cases and on the anterior lens capsule in $28 \%$ cases. Mean intraocular pressure was $18.63 \pm 3.85 \mathrm{~mm}$ of $\mathrm{Hg}$.

During the study period, 1382 cataract surgeries were performed but after fulfilling the inclusion and exclusion criteria 150 patients were selected for the study. The study group consisted of $64 \%$ males and $36 \%$ females having mean age of $65 \pm 15.32$ years respectively.

During surgery, difficulty was encountered in $58.6 \%$ of the cases. The common difficulty encountered was poor pupil dilatation, which was seen in $37.3 \%$ cases (table 1). $19.3 \%$ of the patients required pupillary sphincterotomy for nucleus delivery. Posterior capsular rupture occurred in 5.3\%, $4 \%$ cases were converted to extracapsular cataract extraction and $2.6 \%$ were implanted with anterior chamber IOL.

On the first post-operative day, residual lens matter was noted in $2.6 \%$ of the study group. Other complications are shown in table 2.

Table 1: Intraoperative Complications of MSCS in Eyes with PEX.

\begin{tabular}{lc}
\hline Complications & $\mathbf{n = 1 5 0}$ \\
\hline Poor pupil dilatation & $56(37.3 \%)$ \\
Zonular dehiscence & $11(7.3 \%)$ \\
Posterior capsular rupture with vitreous prolapse & $8(5.3 \%)$ \\
Conversion to extracapsular extraction & $6(4 \%)$ \\
Anterior chamber IOL implantation & $4(2.6 \%)$ \\
Button hole & $1(0.6 \%)$ \\
Nucleus drop & $1(0.6 \%)$ \\
Iridodialysis & $1(4 \%)$ \\
Total & $88(58.6 \%)$ \\
\hline
\end{tabular}

Table 2: Early Postoperative Complications of MSCS in Eyes with PEX.

\begin{tabular}{ll}
\hline Complications & n = 150 \\
\hline Residual lens matter & $4(2.6 \%)$ \\
IOL decentration & $4(2.6 \%)$ \\
Up drawn pupil with vitreous in anterior chamber & $3(2 \%)$ \\
Shallow anterior chamber & $2(1.3 \%)$ \\
\hline
\end{tabular}

\section{DISCUSSION}

Incidence of pseudo-exfoliation has been reported from $1.87 \%$ to $21 \%$ in different ethnicities but researchers have concluded that the frequency of PEX increases with increasing age. ${ }^{4}$ Rao et al reported in their study that $75 \%$ of PEX patients were of 70 years and above. ${ }^{7}$ He found that PEX prevalence was $2.6 \%$ in less than 70 years but increased to $13.04 \%$ after 70 years. Yalaz et al reported incidence of PEX in $11.2 \%$ after 60 years. $^{8}$ Similarly in an epidemiological study, Erkayhan et al found PEX in mean age of $74.64 \pm 6.88$ years. ' In our study, PEX was seen in $8 \%$ of the patients and more after 60 years of age.

In our study, male to female ratio was 16:9. There are conflicting reports about sex predilection of PEX in literature. Some researchers have shown male preponderance $^{10}$ while Arvind et $\mathrm{al}^{11}$ showed no sex predilection. $^{10,11}$ Avramides reported a female preponderance. ${ }^{12}$

Literature shows that cataract surgery in PEX is a challenging procedure as the PEX material is deposited on the lens, the trabecular meshwork and the pupil leading to frequent problems during cataract surgery. This comprises of intraoperative complications like corneal edema, zonular weakness and posterior capsule dehiscence with vitreous loss. The most frequent reported intraoperative problem is non-dilating or poorly dilating pupil despite instillation of strong mydriatics. Islam et $\mathrm{al}^{3}$ reported that $75 \%$ of PEX patients had poorly dilating pupil whereas Erkayhan et $\mathrm{al}^{9}$ reported $8.98 \%$ eyes with poor pupil dilatation. We also found a significantly high rate of poorly dilating pupils in our study.

In eyes with PEX signs of zonular instability like lens subluxation, zonular dialysis, iridodonesis or phacodonesis should be looked for pre-operatively to avoid intraoperative complications. ${ }^{3,4}$ This zonular instability along with poor dilating pupil make capsulorhexis difficult and there are more chances of posterior capsule rupture and vitreous loss. Erkayhan et $\mathrm{al}^{\mathbf{9}}$ reported a high rate of zonular dialysis, posterior capsular rupture and vitreous loss in PEX eyes as compared to controls $(12.5 \%$ vs. $3.4 \%)$. Islam et $\mathrm{al}^{3}$ did not report such high rates of such complications in PEX. Katsimpris et al reported that posterior capsular rupture occurred in $4.2 \%$ of patients who underwent Phacoemulsification and in $17 \%$ who were operated using extracapsular cataract extraction procedure. ${ }^{13}$ According to him there was a higher rate of vitreous 
loss (17\% vs. $4.2 \%)$ in extracapsular cataract surgery compared to Phacoemulsification but we did not find such high frequency using MSCS. Katsimpris et al recommended that in the presence of zonular weakness careful capsulorhexis should be done and nucleus delivery should be carried out either by doing sphincterectomy or by using iris hooks or retractors. ${ }^{13}$ Furthermore, in order to prevent posterior capsular rupture and vitreous loss in the event of non-dilating pupil, MSCS can be converted into extracapsular cataract extraction and visual restoration can be done by intraocular lens implantation either in the sulcus or the anterior capsular rim and even anterior chamber IOL implantation is possible. ${ }^{14}$ In our study, $4 \%$ of the cases were converted to extracapsular extraction due to posterior capsular rupture.

Intraoperative complications of MSCS reported from previous studies were hyphema and Iridodialysis. ${ }^{3}$ We also came across per-operative complications but hyphema was not found in our study.

Postoperative striate keratopathy and iritis in cataract surgery occurs due to unwarranted intraoperative manipulations and prolong surgery time. Islam et $\mathrm{al}^{3}$ reported iritis in $11.91 \%$ of his cases and $4.49 \%$ corneal decompensation in his study after performing cataract surgery in PEX. Gogate found corneal striate in $7 \%$ cases. $^{15}$ Venkatesh ${ }^{16}$ had $2 \%$ cases of postoperative corneal edema. In our study we found none in our study.

In MSCS, proper wound integrity is to be maintained at the conclusion to prevent shallow anterior chamber. On the first post-operative day, we found shallow anterior chamber in $1.3 \%$ of our cases similar to the study conducted in Rawalpindi which reported it in $1.6 \%$ cases. $^{6}$ A high frequency of postoperative intraocular lens decentration was reported in PEX by Shingleton BJ et $\mathrm{al}^{17}$ but we found it in $2.6 \%$ of our cases.

There are some suggestions to minimize the peroperative problems while performing cataract surgery in PEX eyes. Fontana $\mathrm{L}$ et $\mathrm{al}^{18}$ suggested that sufficient pupil dilation is achievable using a viscoelastic with more dispersion properties or by using iris hooks and pupillary rings. Similarly Hosseini $\mathrm{H}$ et $\mathrm{al}^{19}$ recommended the use of trypan blue in PEX eyes for good staining of the anterior capsule to minimize stress on the zonules during capsulorhexis. For the support of zonular dehiscence and the capsular bag use of capsular tension ring (CTR) has been recommended by Haripriya A et al. ${ }^{20}$

Limitations of our study was that it was a hospital based study which does not truly represent the whole population. Secondly, the preoperative and postoperative visual acuity was not compared. We did not perform corneal pachymetry and did not consider intraocular pressure as a confounding factor. Despite these short-comings this study provides a road map to safety of MSCS in PEX cataract surgery.

\section{CONCLUSION}

We observed that due to poor pupillary dilatation in Pseudoexfoliation, caution should be taken during surgery to minimize capsular rupture. However, with good pupillary dilatation, careful capsulorhexis and minimal stress on the zonules, MSCS can be safely performed in eyes with cataract and PEX.

\section{Ethical Approval}

The study was approved by the Institutional review board/Ethical review board.

\section{Conflict of Interest}

Authors declared no conflict of interest.

\section{REFERENCES}

1. Brajković J, Kalauz-Surać I, Ercegović A, MiletićJurić A, Sušić N, Burić Ž. Ocular pseudoexfoliation syndrome and internal systemic diseases. Acta Clinica Croatica. 2007; 46 (1-Supplement 1): 57-61.

2. Kounsar H, Shaheen N, Rather SR. Epidemiology of pseudoexfoliation syndrome: a hospital based comparative study. Int J Res Med Sci. 2018; 6 (4): 1314.

3. Islam MN, Goswami S, Khanam BSM, Mukherji S. Complications of Cataract Surgery in Patients with Pseudoexfoliation Syndrome in a Tertiary Care Hospital of West Bengal. Int J Sci Stud. 2017; 5 (3): 11-15.

4. Pranathi K, Magdum RM, Maheshgauri R, Patel K, Patra S. A study of complications during cataract surgery in patients with pseudoexfoliation syndrome. J Clin Ophthalmol Res. 2014; 2 (1): 7.

5. Song $\mathbf{P}$, Wang $\mathbf{H}$, Theodoratou $\mathbf{E}$, Chan KY, Rudan I. The national and subnational prevalence of cataract and cataract blindness in China: a systematic review and meta-analysis. J Glob Health, 2018; 8 (1): 010804. 
6. Iqbal Y, Zia S, Baig MAU. Intraoperative and early postoperative complications of manual sutureless cataract extraction. J Coll Physicians Surg Pak. 2014; 24 (4): 252-255.

7. Rao RQ, Arain TM, Ahad MA. The prevalence of pseudoexfoliation syndrome in Pakistan. Hospital based study. BMC Ophthalmology, 2006; 6 (1): 27.

8. Yalaz M, Othman I, Nas K, Eroğlu A, Homurlu D, Cikintas Z, et al. The frequency of pseudoexfoliation syndrome in the eastern Mediterranean area of Turkey. Acta Ophthalmol (Copenh), 1992; 70: 209-213.

9. Erkayhan GE, Dogan S. Cataract Surgery in Patients with Pseudoexfoliation Syndrome. Eurasian J Med. 2017; 49: 22-25.

10. Naik AU, Gadewar SB. Visual Outcome of Phacoemulsification versus Small Incision Cataract Surgery in Pseudoexfoliation Syndrome-A Pilot Study. J Clin Diagn Res. 2017; 11 (1): 5.

11. Arvind H, Raju P, Paul PG, Baskaran M, Ramesh SV, George RJ, et al. Pseudoexfoliation in South India. Br J Ophthalmol. 2003; 87: 1321-1323.

12. Avramides S, Traianidis P, Sakkias G. Cataract surgery and lens implantation in eyes with exfoliation syndrome. J Cataract Refract Surg. 1997; 23: 583-587.

13. Katsimpris JM, Petropoulos IK, Apostolakis K, Feretis D. Comparing phacoemulsification and extracapsular cataract extraction in eyes with pseudoexfoliation syndrome, small pupil, and phacodonesis. Klin Monbl Augenheilkd. 2004; 221 (05): 328-333.

14. Gimbel HV, Sun R, Ferensowicz M, Penno EA, Kamal A. Intraoperative management of posterior capsule tears in phacoemulsification and intraocular lens implantation. Ophthalmology, 2001; 108 (12): 2186-2189.

15. Gogate PM. Small incision cataract surgery: complications and mini-review. Indian J Ophthalmol. 2009; 57: 45-49.

16. Venkatesh R, Das M, Prasanth S, Muralikrishnan R. Manual small incision cataract surgery in eyes with white cataracts. Indian J Ophthalmol. 2005; 53: 173176.
17. Shingleton BJ, Neo YN, Cvintal V, Shaikh AM, Liberman P, O'donoghue MW. Outcome of phacoemulsification and intraocular lens implantion in eyes with pseudoexfoliation and weak zonules. Acta Ophthalmol. 2017; 95 (2): 182-187.

18. Fontana L, Coassin M, Iovieno A, Moramarco A, Cimino L. Cataract surgery in patients with pseudoexfoliation syndrome: current updates. Clin Ophthalmol. 2017; 11: 1377.

19. Hosseini H, Nowroozzadeh MH, Razeghinejad MR, Ashraf H, Salouti R, Ashraf MJ. Anterior lens capsule has more affinity to trypan blue in patients with pseudoexfoliation. Eye, 2011; 25 (9): 1245-1246.

20. Haripriya A, Ramulu PY, Schehlein EM, Shekhar M, Chandrashekharan S, Narendran $\mathbf{K}$, et al. The Aravind Pseudoexfoliation Study (APEX): 5-year PostOperative Results. The Effect of IOL Choice and Capsular Tension Rings. Am J Ophthalmol. 2020.

Doi: 10.1016/ j.ajo.2020.06.031.

\section{Authors' Designation and Contribution}

Yasir Iqbal; Associate Professor: Concepts, Design, Manuscript preparation, Manuscript review.

Qaim Ali Khan; Associate Professor: Design, Literature research, Manuscript editing.

Sohail Zia; Associate Professor: Literature research, Data analysis, Manuscript editing.

Muhammad Usman Arshad Qureshi; Senior Registrar: Data acquisition, Data analysis, Manuscript editing.

Masud-ul-Hassan; Consultant Ophthalmologist: Concepts, Statistical analysis, Manuscript editing. 